\title{
Silicon optical modulators
}

\author{
G.T. Reed, G. Mashanovich, F.Y. Gardes and D. J. Thomson
}

\begin{abstract}
Optical technology is poised to revolutionise short reach interconnects. The leading candidate technology is silicon photonics, and the workhorse of such interconnect is the optical modulator. Modulators have been improved dramatically in recent years. Most notably the bandwidth has increased from the MHz to the multi $\mathrm{GHz}$ regime in little more than half a decade. However, the demands of optical interconnect are significant, and many questions remain unanswered as to whether silicon can meet the required performance metrics. Minimising metrics such as the energy per bit, and device footprint, whilst maximising bandwidth and modulation depth are non trivial demands. All of this must be achieved with acceptable thermal tolerance and optical spectral width, using CMOS compatible fabrication processes. Here we discuss the techniques that have, and will, be used to implement silicon optical modulators, as well as the outlook for these devices, and the candidate solutions of the future.
\end{abstract}

\section{Introduction}

Interconnection in networks, both as a conventional data network, or as intrachip or interchip data links, continue to scale in complexity and bandwidth. The limitations of copper as an interconnect medium in terms of loss, dispersion, crosstalk, and fundamental speed, are increasingly obvious as interconnect density is increased. This has heralded the emergence of the optical interconnect, most notably silicon photonics, which has emerged as the leading candidate due to the unique combination of potential low cost, performance enhancements due to the prospect of both photonic, and electronic/photonic integration, as well as compatibility with the world's most successful electronic production technology, CMOS.

Over time, a transition from electrical to optical technology will emerge in the interconnect market, the longer of the short links, which require larger bandwidths will, to begin with, be most feasible to displace. The penetration of optical links into very short distances will then come over time, as the technology becomes more cost effective and with increasing bandwidth requirements. Target interconnects are in application areas such as desktop interconnect, mobile to server interconnect, and data center to high performance computing. 
Whilst the interconnect application is dominant, with active optical cables already reaching the market place, and complex intrachip interconnect the subject of advanced research for high performance computing, there is also a plethora of other application areas that will benefit from the success of silicon photonics. These include fibre-to-the-home (FTTH) or fibre-to-the-premises (FTTP), environmental monitoring, biological and chemical sensing, medical and military applications, astronomy etc. However, the optical interconnect is the hottest topic at the moment due to an urgent application need. Recent bandwidth improvements in electronic interconnects have only been achieved at the expense of increased latency and power consumption [1]. Optical interconnects could bring multiple major advantages compared to their electrical counterparts: they can enable separation of electronic devices, and re-partitioning of the platform whilst retaining high data rates. They can also introduce more conventional advantages of optical technology such as the reduction of EMI, cables and weight, as well as bringing more complex design by introducing enabling modules and optimised cooling . Finally they can relax interconnect density, save energy, and retain precise clock and signal timing [2].The potential energy saving is important, as the predicted amount of heat that can be removed from a chip, in a cost-effective manner, remains flat in the foreseeable future [3]. Since the interconnect will consume $80 \%$ of the microprocessor power [3], and server interconnect power exceeds the total power generated from solar energy [4], optical interconnects can potentially offer substantial energy savings [5], [6].

The ultimate physical manifestation of the silicon photonic device would be as part of an optoelectronic integrated circuit (OEIC) formed monolithically in silicon, combining the photonic functionality and the electronic intelligence in seamless integration. Whilst components continue to be developed, interim solutions include hybrid combinations of different materials to fulfil the functions that silicon is, as yet, unable to deliver. The question remains as to whether silicon will ever deliver all of the components of such a circuit in monolithic form, or whether a hybrid solution will win out. The former would be more elegant, but may never be realisable as a commercial success, due to the ferocious performance demands of the market, at continually reducing cost. In this paper we discuss the progress of just one of the components that will form the OEIC of the future, the silicon optical modulator. Even this single component has been realised both in monolithic and hybrid form, and it is hoped that the discussion provided here will stimulate further debate on the relative merits of each approach.

For any interconnect solution, optical modulation is one of the main functionalities required in a photonic circuit. An optical source can be either directly or externally modulated. External modulation offers several 
advantages compared to direct modulation: the optical source can be relatively inexpensive and its operation does not need to be compromised by direct modulation, modulation speed can be higher, and direct phase modulation is possible [7] and optical isolation and wavelength stabilization need be done only once for the whole system.. Furthermore, a single light source can feed multiple channels via individual modulators, reducing the total power budget of any given system.

\section{Modulation in silicon}

An optical modulator is a device that is used to modulate a light beam that propagates either in free space or in an optical waveguide. This device can alter different beam parameters and therefore optical modulators can be categorized as amplitude, phase, or polarisation modulators. In addition, modulators can be also classified into two operational categories: electrorefractive and electroabsorptive.

The application of an electric field to a material can result in a change to the real and imaginary refractive indices. A change in real refractive index, $\Delta \mathrm{n}$, with an applied electric field is known as electrorefraction, and a change in the imaginary part of refractive index, $\Delta \alpha$, with applied electric field is known as electroabsorption. The primary electric field effects that are traditionally useful in semiconductor materials to cause either electroabsorption or electrorefraction are the Pockels effect, the Kerr effect, and the FranzKeldysh effect. However, it has been shown that these effects are weak in pure silicon at the communications wavelengths of $1.3 \mu \mathrm{m}$ and $1.55 \mu \mathrm{m}[8,9]$. Therefore alternative modulation means have to be found for silicon. One option is to use thermal modulation, as silicon exhibits a large thermo-optic coefficient, although this is too slow for the high frequencies required in modern communications applications [10].

The most common modulation method used in silicon devices to date is the plasma dispersion effect, in which the concentration of free charges in silicon changes the real and imaginary part of the refractive index [9]. Soref and Bennett [8] studied results in the scientific literature to evaluate the change in refractive index, $\Delta \mathrm{n}$, due to experimentally produced absorption curves for a wide range of electron and hole densities, over a wide range of wavelengths. They also quantified the changes that they had identified from the literature, for both changes in refractive index and in absorption [8]. They produced the following expressions to evaluate changes in the density of carriers in silicon:

At $\lambda_{0}=1.55 \mu \mathrm{m}:$ 
$\Delta \mathrm{n}=\Delta \mathrm{n}_{\mathrm{e}}+\Delta \mathrm{n}_{\mathrm{h}}=-\left[8.8 \times 10^{-22} \cdot \Delta \mathrm{N}_{\mathrm{e}}+8.5 \times 10^{-18} \cdot\left(\Delta \mathrm{N}_{\mathrm{h}}\right)^{0.8}\right]$

$\Delta \alpha=\Delta \alpha_{\mathrm{e}}+\Delta \alpha_{\mathrm{h}}=8.5 \times 10^{-18} \cdot \Delta \mathrm{N}_{\mathrm{e}}+6.0 \times 10^{-18} \cdot \Delta \mathrm{N}_{\mathrm{h}}$

where:

$\Delta \mathrm{n}_{\mathrm{e}}=$ change in refractive index resulting from change in free electron carrier concentrations.

$\Delta \mathrm{n}_{\mathrm{h}}=$ change in refractive index resulting from change in free hole carrier concentrations.

$\Delta \alpha_{\mathrm{e}}=$ change in absorption resulting from change in free electron carrier concentrations.

$\Delta \alpha_{\mathrm{h}}=$ change in absorption resulting from change in free hole carrier concentrations.

Similarly at $\lambda_{0}=1.3 \mu \mathrm{m}$ :

$\Delta \mathrm{n}=\Delta \mathrm{n}_{\mathrm{e}}+\Delta \mathrm{n}_{\mathrm{h}}=-\left[6.2 \times 10^{-22} \cdot \Delta \mathrm{N}_{\mathrm{e}}+6.0 \times 10^{-18} \cdot\left(\Delta \mathrm{N}_{\mathrm{h}}\right)^{0.8}\right]$

$\Delta \alpha=\Delta \alpha_{\mathrm{e}}+\Delta \alpha_{\mathrm{h}}=6.0 \times 10^{-18} \cdot \Delta \mathrm{N}_{\mathrm{e}}+4.0 \times 10^{-18} \cdot \Delta \mathrm{N}_{\mathrm{h}}$

Using these expressions, it is straightforward to calculate that a change in the carrier density of the order of $5 \times 10^{17} \mathrm{~cm}^{-3}$ results in a change in refractive index, $\Delta \mathrm{n}$, of $-1.66 \times 10^{-3}$, at a wavelength of $1.55 \mu \mathrm{m}$, for example. However, the change in refractive index is necessarily accompanied by a detrimental change in intensity due to the absorption via free carriers [11].

Electrical manipulation of the charge density interacting with the propagating light is achievable through mechanisms such as carrier injection, accumulation or depletion. These schemes are represented schematically in waveguide cross sections in figure 1.

More recently, attempts have been made to investigate alternative modulation mechanisms in other materials potentially compatible with silicon technology, such as germanium, in order to produce more efficient modulation. The Franz-Keldysh effect [12] and the quantum confined Stark effect (QCSE) are electric field induced changes in optical absorption.. They are related, with the former being the limit of the latter as the quantum-well layers are made thicker. The QCSE has more spectrally abrupt and stronger changes in absorption coefficient [4]. It is observed in quantum well structures where the electron and hole confinement afforded by the barriers allows for exciton enhancement of the optical absorption. However, to utilise these effects on a silicon platform necessitates the complication of introducing a second material. In 2005, Kuo et al demonstrated the QCSE with pure Ge quantum wells and Ge-rich SiGe barriers on Si [13]. Similar 
structures exhibiting the QCSE were later integrated into modulator devices, demonstrating proof-of-concept Electro Absorption Modulation (EAM) [14, 15], although no waveguide based device have yet been reported. Nevertheless this work offers significant hope that high speed, efficient QCSE modulators may emerge in the future, albeit with more complex fabrication requirements.

Further modulation options are available through the hybridisation of silicon with other photonic materials or the manipulation of the 'standard' crystalline material; these will be discussed briefly in the state of the art section of this paper.

So far in this text we have discussed how optical modulators in silicon can vary the refractive index of the material, or how to induce absorption. The latter directly modulates the intensity of a propagating mode, so it is obvious how modulation is achieved in such devices. However, in order to convert a refractive index change into intensity modulation, essentially two options are available to the designer. Firstly the refractive index change can be used to shift the relative phase of two propagating waves to either interfere constructively or destructively. Typically, Mach Zehnder interferometers are utilised for this. Secondly, resonant structures can be used, and the refractive index change induced in the modulator can be used to change the resonant condition such that the device switches between on and off resonance at any given wavelength. Discussion of the relative merits of each approach will be included in the next section which considers modulator performance metrics.

\section{Performance Metrics}

There are several figures of merit that are used to characterise a modulator: modulation speed, modulation depth, optical bandwidth, insertion loss, area efficiency (footprint) and power consumption.

The modulation speed/bandwidth is one of the most important figures of merit for an optical modulator. The modulation bandwidth is usually defined by the frequency where the modulation is reduced to $50 \%$ of its maximum value. The speed of a modulator is also commonly characterised by its ability to carry data at a certain rate. High modulation speeds are required for interconnect applications where high data rates are imperative, whilst for some other applications (e.g. sensing) only relatively moderate modulation speeds are needed. 
Modulation depth is defined as the ratio of two intensities: $I_{\max }$ - the intensity transmitted when the modulator is adjusted for maximum transmission and $\mathrm{I}_{\min }$ - the intensity transmitted when the modulator is adjusted for minimum transmission. It is quoted in decibels and expressed as: $10 \log \left(\mathrm{I}_{\max } / \mathrm{I}_{\min }\right)$. A large modulation depth is useful for long transmission distance, good bit error rate, and improved receiver sensitivity. However, whilst a high modulation depth or extinction ratio $(>7 \mathrm{~dB})$ is always preferred; when taking into account the overall system link budget and trading of the system metrics; for "interconnect " applications, an extinction ratio in the region of 4 to $5 \mathrm{~dB}$ is sufficient. However, the performance metrics of an interconnect system are linked in a relatively simple way, such that it is possible to trade off performance in one area for another. For example, a shorter modulator will result in inferior extinction ratio, but reduced power consumption, and reduced footprint. Consequently it is possible to optimise performance for a given application depending on the dominant metric.

Insertion loss takes into account the optical power lost when the modulator is added into the photonic circuit. It is a passive loss which comprises reflection, absorption, and mode coupling losses. The insertion loss is significant as it contributes to the link budget set by the receiver sensitivity and overall losses in the end to end system. Whilst there has been a trend in silicon photonics to move to smaller sub micrometer waveguides, largely fuelled by device performance enhancements and compactness, this move has been coupled with an increase in loss mainly due to coupling loss and a greater modal interaction with sidewall roughness. Performance enhancements in small cross section devices should therefore be evaluated against any loss increase

In addition to the 'standard' metrics of optical modulators previously mentioned, power consumption has become particularly important when optical interconnects are considered: i.e. the energy expended in producing each bit of data, or the "energy per bit", (although the term "power per bit" is also used colloquially to mean the same thing). In order to justify the introduction of optical interconnects, their power consumption cannot exceed that of the current electrical interconnects, which means that future systems needs to target less than $1 \mathrm{pJ} / \mathrm{bit}[16]$, and arguably needs to offer a substantial reduction in power/energy consumption, although this is an extremely demanding target. Some authors argue that this means that the total (on-chip) system energy needs to target $\sim 100 \mathrm{fJ} /$ bit to exceed the efficiency of the equalized on-chip electrical link at the $45 \mathrm{~nm}$ process node [17]. Since there are several energy contributions in this power budget, the energy per bit for an optical output device (modulator in this case) needs to be $\sim 10 \mathrm{fJ} / \mathrm{bit}[18]$. On the other hand, electrical I/O's alone currently consume 2-3 pJ/bit, Whilst power consumption is important 
the added value that optical interconnect brings could justify a larger power consumption. Note that today typical electrical consumption of servers is of the order of 10-30pJ/bit [5] and hence one may not necessarily need an order or magnitude improvement to justify value. Similarly typical commercial 10Gpit/s VCSEL optical links have an end to end power consumption of the order of $10 \mathrm{pJ} / \mathrm{bit}$ [19]. Therefore some of the theoretical figures discussed in the literature for power consumption are extremely demanding for state of the art silicon modulators. For example, one of the most compact MZI modulators reported so far has an energy requirement of $5 \mathrm{pJ} / \mathrm{bit}$ at $10 \mathrm{~Gb} / \mathrm{s}$ for a $\sim 200 \mu \mathrm{m}$ long device [20]. Clearly this is still much larger than the target of $\sim 10 \mathrm{fJ} / \mathrm{bit}$ proposed above, yet it may bring significant advantages. Electroabsorption modulators could potentially offer low operating energies as the QCSE effect is strong, they are not based on changing the carrier density and they have been demonstrated with CMOS compatible driving voltages [21]. They are promising for both waveguide and free-space optical systems.

Another important feature of a device is the footprint. MZI based modulators tend to require a long interaction length for a complete transition between a maximum and a minimum ( mm's). This hinders high speed performance and results in greater insertion loss, cost and power consumption. The footprint of resonant devices on the other hand can typically be much smaller (e.g. [22-26] ). Therefore this discussion might understandably have led to the conclusion that resonant devices are superior over those based in MZI structures. However, there are further factors to take into account which encourage discussion.

The optical bandwidth refers to the useful operational wavelength range of the device. Resonant based devices tend to function over a relatively narrow band in comparison to MZI based modulators and whilst significant footprint savings are obtainable the resultant bandwidth reduction can go from more than $20 \mathrm{~nm}$ in the case of a MZI to around $100 \mathrm{pm}$ in the case of ring resonators [24].

Narrow band devices are limited in the applications for which they are compatible but perhaps their main shortcoming is their sensitivity to typical fabrication tolerances and to temperature variations. Considering the effect that fabrication tolerances can have, in ring resonators for example the resonant frequency shifts by approximately $0.25 \mathrm{~nm}$ for every nanometer increase in average ring waveguide width [17]. To hit the right resonant wavelength can be challenging using the $130 \mathrm{~nm}$ CMOS generation as the $3 \sigma$ fabrication tolerance is $40 \mathrm{~nm}$. This figure is significantly reduced with the implementation of $45 \mathrm{~nm}$ or $22 \mathrm{~nm}$ CMOS generations, to 16 and $1.2 \mathrm{~nm}$, respectively [27]. In order to overcome this limitation, less fabrication 
sensitive rings in SiN have been investigated recently [28], as well as eight-channel second order microringresonator filter banks with accurate spectral spacing using modified scanning-electron-beam lithography [29]. The sensitivity of a symmetrically configured MZI to global temperature changes is theoretically very small, depending only on variations in the characteristics of the coupler structure. Ring resonators on the other hand are extremely temperature sensitive $(30-80 \mathrm{pm} / \mathrm{K}$ [30], [31] due to the large thermo-optic coefficient of silicon [9], and the narrow resonant spectral width $(\sim 0.1 \mathrm{~nm}$ for single ring [32] and 0.5-2 $\mathrm{nm}$ for higherorder rings [33],[34] This means that temperature stabilisation is needed [35] which requires thermo electric coolers and controllers increasing power consumption. To emphasise this issue let's take the example of a typical server/pc system operating within a temperature range of $70{ }^{\circ} \mathrm{C}$. If a ring resonator modulator was to be used in such a system it would have to be held within the order of $1^{\circ} \mathrm{C}$ to operate to specifications. This would involve the need to power a Thermo Electric Cooler (TEC) to obtain the required temperature control. The extra power consumption due to temperature controller, together with the continuous temperature drift if the device was to be packaged next to a CPU would negate the advantage of the power consumption gained in using such temperature sensitive device. Hence the design must mitigate against temperature drift, which in turn has yielded studies to minimise temperature dependence by incorporating claddings with a temperature dependence with the opposite sign to that of silicon [30],[36]. Currently this approach is not a CMOS compatible process. Multiple rings can also provide low temperature drift although over a relatively small wavelength range [37]. Recently, passive temperature compensation has been demonstrated by using a ring coupled to a Mach-Zehnder interferometer [38]. Electroabsorption modulators, on the other hand, are likely to be much less temperature sensitive than microring resonator refractive devices [4].

In many cases, the metrics discussed above are involve trade-offs. For example, the footprint of a Mach Zehnder interferometric modulator can be reduced, which also reduces the power consumption, and the optical and RF losses. However, the reduced phase shift means that the interferometer null is less deep as the two waves in the interferometer are not fully in antiphase, and hence the modulation depth is reduced [39]. Similarly the optical bandwidth of a ring resonator and its thermal stability can be improved by cascading multiple rings but disadvantages of this method are the added complexity, larger footprint and power consumption, and increased channel spacing [34].

Another possibility to reduce footprint is the so called slow light approach. Slow light is a resonance effect resulting from the light propagating in a specific periodic structure. In these structures the light is coherently scattered and interferes with the incoming light, forming an interference pattern that moves forward slowly. 
Such periodic structures can be found in a form of corrugated waveguides or photonic crystals. Depending on the structure or periods being used, for the slow light effect to be useful for modulation, it is necessary to operate at wavelengths away from those satisfying the Bragg condition where standing waves turn the device into a high reflectivity mirror. When the conditions are met, slow light modulators operate by slowing the effective velocity of the passing light, hence the interaction with the modulating media can be enhanced, resulting in a device length or drive voltage reduction. These devices however, operate again via a resonant effect and consequently optical bandwidth limitations apply.

Whilst the intention in this paper is to discuss the impact and performance of silicon based optical modulators, their advantages cannot be divorced from the added value of silicon photonics in facilitating optical integration at low cost. The modulator is integral to such advantage, since a high aggregate data rate system can only be implemented in a high volume application in integrated format. Silicon photonics allows such implementation by facilitating combinations of high data rate modulators into a high aggregate bandwidth system.

\section{The state of the art}

Research into silicon waveguide based optical modulators dates back to the mid 1980's [40]. In the early years, devices were generally slow $(\sim \mathrm{MHz})$ and seemingly had little prospect for applications in high speed systems. Most monolithic devices employed carrier injection, using the plasma dispersion effect for either modulation of the material refractive index or its absorption [40-59]. In most cases PIN diode structures were formed around the waveguide to electrically control the injection of electrons and holes into the path of the propagating light. To avoid excessive optical loss, the waveguides were positioned in the intrinsic region of the diode and the doped regions positioned in such a way that modal overlap was minimal. Performance enhancements were progressively made through the years by optimising the structure and reducing device dimensions and by the mid 2000's device bandwidths of around the GHz regime were being proposed [58]. One such device by Png et al. is shown schematically in figure 2(a).

In 2004 the breakthrough came when the first monolithic silicon modulator capable of operation at speeds in excess of $1 \mathrm{GHz}$ was experimentally demonstrated [60]. The device, as shown in figure 2(b), functioned by accumulating free carriers on either side of a dielectric layer, inside a micrometer sized waveguide, much like in a capacitor, rather than by carrier injection. 
The performance of this device was later optimised and data rates of $10 \mathrm{~Gb} / \mathrm{s}$ were achieved with an extinction ratio of $3.8 \mathrm{~dB}$ [61]. Since the unveiling of this device other accumulation based modulators have been reported [62], most notably the device recently demonstrated by Lightwire which achieved $10 \mathrm{~Gb} / \mathrm{s}$ with an extinction ratio of $9 \mathrm{~dB}$ as shown in figure 2(e) [63]. It is also interesting to point out that the efficiency of this device demonstrated an improvement of almost an order of magnitude as compared to [61], enabling an active length of about 480 microns rather than $3.45 \mathrm{~mm}$ in the case of [61].

The dynamic operation of carrier accumulation modulators is not limited by the relatively long minority carrier lifetime in silicon, which is the case with carrier injection, but is dominated by the device resistance and capacitance. A third technique for manipulating free carrier densities in the device which also removes the minority carrier lifetime limitation is carrier depletion. Such devices operate by interaction of the propagating light with the junction region of a pn diode which is operated with a reverse bias. The diode's depletion width and therefore free carrier density in the waveguide is varied with applied reverse bias. In 2005 Gardes et al. proposed the first modultor using this technique in a submicrometer waveguide, as shown in figure 3(a). Modelling data predicted an unprecidented intrinsic bandwidth of $50 \mathrm{GHz}$ [64]. The device featured a horizontal pn junction across the waveguide with the highly doped regions required for the formation of resistive contacts placed at the extremities of the waveguide to avoid excessive optical loss.

Two years later a design similar to that proposed by Gardes et al. was realised by Liu et al. [65] . The authors first reported data transmission at $30 \mathrm{~Gb} / \mathrm{s}$ [65] but later improved this figure, demonstrating data transmission at $40 \mathrm{~Gb} / \mathrm{s}$, as shown in figure 3@ [39]. Further to this a plethora of carrier depletion based modulators compatible with data transmission at speeds of at least $10 \mathrm{~Gb} / \mathrm{s}$ were demonstrated [23-25, 6673]; but the device from Liu et al. [39] is still the fastest silicon modulator reported to date. The different devices described above demonstrate the possibility of achieving a high modulation efficiency using an injection device but at the expense of the modulation speed. The opposite is true for depletion devices where a modulation speed in excess of $30 \mathrm{GHz}$ can be reached, but at reduced efficiency. The challenge remains to improve efficiency whilst maintaining other performance metrics.

In the dicussion so far, two of the main metrics considered in respect of reported devices have been the modulation speed (bandwidth) and the area efficiency of the devices. As the speed limitation was seemingly becoming increasingly surmountable, requirements for the enhancement of other performance metrics grew in importance such as for example, power consumption and device footprint. 
The reduction in device footprint and therefore power consumption achievable through the use of resonant structures has already been discussed. Active high speed ring resonators were first introduced by Xu et al. in 2005 [22]. This device is shown schematically in figure 4(a). The authors introduced a PIN diode, carrier injection scheme into the submicron waveguide based ring resonator structures, achieving at first, a data rate of $1.5 \mathrm{~Gb} / \mathrm{s}$. The rings had a diameter of $12 \mu \mathrm{m}$ so clearly the footprint of this device is much less than the MZI modulators reported thus far. The authors later reported improved results achieving $16 \mathrm{~Gb} / \mathrm{s}$ with an 8 $\mathrm{dB}$ extinction ratio by using a pre-emphasis driving signal [74]. This method of modulation was also employed by Green et al. in a MZI type modulator [20] where a data rate of $10 \mathrm{~Gb} / \mathrm{s}$ was reported for a length of 200 microns. The use of pre-emphasis drive signals was first proposed in silicon by Png et al. in 2004 [75] and involves manipulation of the drive signal shape to reduce state transition times [58]. Resonant based modulators have also been made using carrier depletion structures where bandwidths compatible with data rates of $10 \mathrm{~Gb} / \mathrm{s}$ have been demonstrated using a standard square wave drive signal [23-26]. The fastest ring resonator carrier depletion based structure reported to date operates at a bandwidth greater than $35 \mathrm{GHz}$ $[72]$.

As far as slow-light devices are concerned, photonic crystal based MZI devices were first reported by Soljacic et al. [76] and replicated by other researchers [77-80] using temperature modulation or through the injection or accumulation of free carriers. One of the main advantages of these devices is the ability to engineer the group velocity to enable device lengths in the order of 100 microns but this can be at the cost of optical bandwidth as previously discussed [80]. In order to improve the bandwidth, the dispersion engineering technique can be used [81] to achieve operating bandwidths of $10 \mathrm{~nm}$ which provide temperature insensitivity over a much wider range. Nevertheless modulation speeds of only about $1 \mathrm{GHz}$ have been reported to date in these structures in silicon. Corrugated waveguide modulators have also been proposed using the same effect [82].

The power consumption of devices has been discussed more and more in recent years. Modulators based on microring resonators detailed in recent papers by the Kotura and Cornell groups have reported 50 fJ/bit @ 5 Gb/s and 86 fJ/bit @ 3 Gb/s, respectively [24], [83]. However, as mentioned above, microrings are very temperature dependent and therefore temperature stabilization may be required. If $1 \mathrm{~mW}$ is required for the ring temperature stabilisation at $10 \mathrm{~Gb} / \mathrm{s}$, this would represent an additional $100 \mathrm{fJ} / \mathrm{bit}$. Simulations performed by Batten et al. showed that the heating budget for the whole optical link should not exceed 100 
fJ/bit [84]. By taking the temperature stabilisation into account, the MIT group reported $\sim 300 \mathrm{fJ} / \mathrm{bit}$ for a modulation scheme [85], again significantly higher than the proposed target. By using flip-chip integration and employing a heat sink on top of the modulator, the first sub-picojoule per bit (400 fJ/bit) silicon modulator integrated with a driver circuit and embedded in a clocked digital transmitter has been reported recently [86]. The modulator alone, consumed $120 \mathrm{fJ} / \mathrm{bit}$. The authors stated that with no active temperature control employed, they maintained error-free operation with a bit-error-rate lower than $10^{-15}$ at a data rate of $5 \mathrm{~Gb} / \mathrm{s}$. A waveguide Franz-Keldysh modulator with an estimated power per bit of $50 \mathrm{fJ} / \mathrm{bit}$ has also been recently demonstrated [12].

So far this review has predominantly discussed monolithic modulators which use the plasma dispersion effect for operation, together with a brief discussion of Franz-Keldysh, thermal, and QCSE modulators. Some key devices that have been discussed together with their performance metrics are highlighted in table 1. Alternatives do exist, but at the expense of CMOS compatibility or fabrication complexity. Arguments for a hybrid modulator are perhaps reinforced if one accepts that other devices such an optical source or detector can also be usefully integrated via a hybrid approach, which to date has most successfully provided the most efficient light source in silicon by the introduction of III-V material onto the silicon waveguide. Debate still rages as to whether integration of the light source is even required on chip, but there is no doubt that if one hybrid component is required, others can be included at incidental additional cost. Modulators formed in this way have been demonstrated [87-89] with the most recent advances achieving a data rate of $25 \mathrm{~Gb} / \mathrm{s}$ at a 10 $\mathrm{dB}$ extinction ratio [89]. Modulation via the Kerr effect was demonstrated at $10 \mathrm{GHz}$ [90], although the use of an engineered optical polymer waveguide cladding was required to overcome the relatively weak nonlinearities in silicon. As previously discussed SiGe modulators which employ the QCSE [13, 91] (figure 5) and Franz-Keldysh effect [92] have also been reported, and modulators fabricated in amorphous silicon may find employment in low temperature back end integration schemes [93]. Electro-absorption modulation has been demonstrated in two multi-stack structures, based on a-Si:H/a-SiCN [94]. The introduction of strain to crystalline silicon has also been shown to induce the otherwise absent Pockels effect [95], which could provide a further modulation option in the future. However, this effect needs further investigation as the theoretical coefficient is significantly smaller than those experimentally reported by one model [96].

\section{The outlook}

The development of optical modulators in silicon has clearly gone through a period of rapid improvement in the past 6 years. Commercial products and devices, integrated with various other photonic/electronic 
components are beginning to emerge in niche areas such as active optical cables [63]. These devices utilise multiplexed modulators operating at $10 \mathrm{~Gb} / \mathrm{s}$ rather than using singular $40 \mathrm{~Gb} / \mathrm{s}$ devices, a decision which was surely motivated by the impractical extinction ratio achieved to date at the latter data rate, as well as the ease with which suitable drive electronics can be integrated.

Ideally, optical modulators are required that have high modulation speed, large bandwidth, and small footprint, as well as low loss and ultra low power consumption. They also need to be CMOS compatible. These requirements, however, often contradict one another, and therefore an innovative engineering solution is necessary to achieve an optimal trade-off. This means that despite impressive results recently achieved in this field, there are still significant challenges that the silicon photonics research community needs to overcome in the future, before a full deployment of the technology in several important application areas becomes a reality. Nevertheless, silicon photonics offers one of the most promising approaches to achieving high density electronic/photonic integration that is necessary to develop a range of applications, most notably optical interconnect for high performance computing.

The question remains as to whether the OEIC will be realised as a monolithic or hybrid circuit. In some ways this question is merely a diversion, because any technology develops dynamically. The overwhelming evidence is that silicon photonics has now developed such significant momentum, and promises a sufficiently viable technological solution, that implementation is almost inevitable, regardless of the precise detail of the ultimate commercially successful platforms. As for the modulator, the monolithic variants continue to improve, but it is entirely possible, given the wealth of expertise currently devoted to this subject, that a novel device design could change the landscape overnight. Whether this device is monolithic or hybrid remains to be seen, and in the opinion of the authors is largely immaterial. Much like the OEIC, the final form of the technology is unimportant. What we do know is that Silicon Photonics will make a difference, and is here to stay.

[1] David J. Miller, Philip M. Watts, and Andrew W. Moore, "Motivating Future Interconnects: A Differential Measurement Analysis of PCI Latency " in ACM/IEEE Symposium on Architectures for Networking and Communications Systems Princeton, New Jersey, USA, 2009, pp. 19-20 October

[2] D. A. B. Miller, "Rationale and challenges for optical interconnects to electronic chips," Proceedings of the IEEE, vol. 88 , pp. $728-749,2000$.

[3] ITRS, " www.itrs.net/links/2007itrs/execsum2007.pdf," 2007.

[4] D. Miller, "Device Requirements for Optical Interconnects to Silicon Chips," Proceedings of the IEEE, vol. 97, pp. 1166-1185, 2009.

[5] P. Pepeljugoski, J. Kash, F. Doany, D. Kuchta, L. Schares, C. Schow, M. Taubenblatt, B. J. Offrein, and A. Benner, "Low power and high density optical interconnects for future supercomputers," Optical Fiber Communication Conference (OFC), San Diego, California, 21 March 2010, paper OThX2

[6] B. G. Lee, A. Biberman, J. Chan, and K. Bergman, "High-Performance Modulators and Switches for Silicon Photonic Networks-on-Chip," Selected Topics in Quantum Electronics, IEEE Journal of, vol. 16, pp. 6-22, 2010.

[7] C. Pollock and M. Lipson, Integrated Photonics. Boston: Kluwer Academic Publishers, 2003. 
[8] R. A. Soref, and B.R. Bennett, "Electrooptical Effects in Silicon," IEEE J. Quantum Elect., vol. QE-23, pp. 123-129, January 1987.

[9] G. T. Reed and A. P. Knights, Silicon Photonics: An Introduction: John Wiley \& Sons, Inc., 2004.

[10] G. Cocorullo and I. Rendina, "Thermo-optical modulation at 1.5 microns in silicon etalon," Electronics Letters, vol. 28, pp. 83-85, 1992.

[11] G. T. Reed, Silicon Photonics: The State of the Art: John Wiley \& Sons, Inc., 2008.

[12] J. Liu, M. Beals, A. Pomerene, S. Bernardis, R. Sun, J. Cheng, L. C. Kimerling, and J. Michel, "Waveguide-integrated, ultralow-energy GeSi electro-absorption modulators," Nat Photon, vol. 2, pp. 433-437, 2008.

[13] Y. H. Kuo et al., "Strong quantum-confined Stark effect in germanium quantum-well structures on silicon," Nature, vol. 437, pp. 1334-1336, 2005.

[14] J. E. Roth, O. Fidaner, R. K. Schaevitz, Y.-H. Kuo, T. I. Kamins, J. S. Harris, and D. A. B. Miller, "Optical modulator on silicon employing germanium quantum wells," Opt. Express, vol. 15, pp. 5851-5859, 2007.

[15] J. E. Roth, O. Fidaner, E. H. Edwards, R. K. Schaevitz, Y. H. Kuo, N. C. Herman, T. I. Kamins, J. S. Harris, and D. A. B. Miller, "C-band side-entry Ge quantum-well electroabsorption modulator on SOI operating at $1 \mathrm{~V}$ swing," Electronics Letters, vol. 44, pp. 49-50, 2008.

[16] A. V. Krishnamoorthy, R. Ho, B. O'Krafka, J. E. Cunningham, J. Lexau, and X. Zheng, "Potentials of Group IV Photonics Interconnects for "Red-shift" Computing Applications," in Proc. Group IV Photonics, 2007 pp. 180-182.

[17] T. Barwicz, H. Byun, F. Gan, C. W. Holzwarth, M. A. Popovic, P. T. Rakich, M. R. Watts, E. P. Ippen, F. X. Kärtner, H. I. Smith, J. S. Orcutt, R. J. Ram, V. Stojanovic, O. O. Olubuyide, J. L. Hoyt, S. Spector, M. Geis, M. Grein, T. Lyszczarz, and J. U. Yoon, "Silicon photonics for compact, energy-efficient interconnects," J. Opt. Netw., vol. 6, pp. 63-73, 2007.

[18] S. J. B. Yoo, "Future prospects of silicon photonics in next generation communication and computing systems," Electronics Letters, vol. 45, pp. 584-588, 2009.

[19] A. Alduino and M. Paniccia, "Wiring electronics with light," Nature Photonics, vol. 1, pp. 153-155, 2007

[20] W. M. J. Green, M. J. Rooks, L. Sekaric, and Y. A. Vlasov, "Ultra-compact, low RF power, 10 Gb//s silicon MachZehnder modulator," Opt. Express, vol. 15, pp. 17106-17113, 2007.

[21] Helman, C. Noah, Roth, E. Jonathan, Bour, P. David, Altug, Hatice, and Miller, A. B. David, Misalignment-tolerant surface-normal low-voltage modulator for optical interconnects vol. 11. New York, NY, ETATS-UNIS: Institute of Electrical and Electronics Engineers, 2005.

[22] Q. Xu, B. Schmidt, S. Pradhan, and M. Lipson, "Micrometre-scale silicon electro-optic modulator," Nature, vol. 435, pp. 325-327, 2005.

[23] F. Y. Gardes, A. Brimont, P. Sanchis, G. Rasigade, D. Marris-Morini, L. O'Faolain, F. Dong, J. M. Fedeli, P. Dumon, L. Vivien, T. F. Krauss, G. T. Reed, and J. Martí, "High-speed modulation of a compact silicon ring resonator based on a reverse-biased pn diode," Opt. Express, vol. 17, pp. 21986-21991, 2009.

[24] P. Dong, S. Liao, D. Feng, H. Liang, D. Zheng, R. Shafiiha, C.-C. Kung, W. Qian, G. Li, X. Zheng, A. V. Krishnamoorthy, and M. Asghari, "Low Vpp, ultralow-energy, compact, high-speed silicon electro-optic modulator," Opt. Express, vol. 17, pp. 22484-22490, 2009.

[25] J.-B. You, M. Park, J.-W. Park, and G. Kim, "12.5 Gbps optical modulation of silicon racetrack resonator based on carrier-depletion in asymmetric p-n diode," Opt. Express, vol. 16, pp. 18340-18344, 2008.

[26] M. R. Watts, D. C. Trotter, R. W. Young, and A. L. Lentine, "Ultralow power silicon microdisk modulators and switches," in Proc. Group IV Photonics, 2008 5th IEEE International Conference on, 2008, pp. 4-6.

[27] Y. Vlasov, "Silicon Photonics for next generation computing systems," 2009.

[28] A. Gondarenko, J. S. Levy, and M. Lipson, "High confinement micron-scale silicon nitride high Q ring resonator," Opt. Express, vol. 17, pp. 11366-11370, 2009.

[29] C. W. Holzwarth, T. Barwicz, M. A. Popovic, P. T. Rakich, E. P. Ippen, F. X. Kartner, and H. I. Smith, "Accurate resonant frequency spacing of microring filters without postfabrication trimming," Journal of Vacuum Science and Technology B, 2006, pp. 3244-3247.

[30] J. Teng, P. Dumon, W. Bogaerts, H. Zhang, X. Jian, X. Han, M. Zhao, G. Morthier, and R. Baets, "Athermal Siliconon-insulator ring resonators by overlaying a polymer cladding on narrowed waveguides," Opt. Express, vol. 17, pp. $14627-14633,2009$.

[31] W. N. Ye, J. Michel, and L. C. Kimerling, "Athermal high-index-contrast waveguide design," Photonics Technology Letters, IEEE, vol. 20, pp. 885-887, 2008.

[32] Q. Xu, D. Fattal, and R. G. Beausoleil, "Silicon microring resonators with 1.5 micrometer radius," Opt. Express, vol. 16, pp. 4309-4315, 2008.

[33] S. Xiao, M. H. Khan, H. Shen, and M. Qi, "A highly compact third-order silicon microring add-drop filter with a very large free spectral range, a flat passband and a low delay dispersion," Opt. Express, vol. 15, pp. 14765-14771, 2007.

[34] F. Xia, M. Rooks, L. Sekaric, and Y. Vlasov, "Ultra-compact high order ring resonator filters using submicron silicon photonic wires for on-chip optical interconnects," Opt. Express, vol. 15, pp. 11934-11941, 2007.

[35] S. Manipatruni, R. K. Dokania, B. Schmidt, N. Sherwood-Droz, C. B. Poitras, A. B. Apsel, and M. Lipson, "Wide temperature range operation of micrometer-scale silicon electro-optic modulators," Opt. Lett., vol. 33, pp. 2185-2187, 2008.

[36] J.-M. Lee, D.-J. Kim, G.-H. Kim, O. K. Kwon, K.-J. Kim, and G. Kim, "Controlling temperature dependence of silicon waveguide using slot structure," Opt. Express, vol. 16, pp. 1645-1652, 2008.

[37] Y. Vlasov, W. M. J. Green, and F. Xia, "High-throughput silicon nanophotonic wavelength-insensitive switch for onchip optical networks," Nat Photon, vol. 2, pp. 242-246, 2008.

[38] B. Guha, B. B. C. Kyotoku, and M. Lipson, "CMOS-compatible athermal silicon microring resonators," Opt. Express, vol. 18, pp. 3487-3493.

[39] L. Liao, A. Liu, D. Rubin, J. A. B. J. Basak, Y. A. C. Y. Chetrit, H. A. N. H. Nguyen, R. A. C. R. Cohen, N. A. I. N. Izhaky, and M. A. P. M. Paniccia, "40 Gbit/s silicon optical modulator for highspeed applications," Electronics Letters, vol. 43, 2007.

[40] R. A. Soref and B. R. Bennett, "Kramers-Kronig analysis of electro-optical switching in silicon," in Proc.SPIE: Integrated Optical Circuit Engineering IV, 16-17 Sept. 1986, Cambridge, MA, USA, 1987, pp. 32-7.

[41] J. P. Lorenzo and R. A. Soref, "1.3 mm electro-optic silicon switch," Applied Physics Letters, vol. 51, pp. 6-8, 1987.

[42] L. Friedman, R. A. Soref, and J. P. Lorenzo, "Silicon double-injection electro-optic modulator with junction gate control," J. Appl. Phys., vol. 63, pp. 1831-9, 1988/03/15 1988. 
[43] G. V. Treyz, "Silicon Mach-Zehnder waveguide interferometers operating at $1.3 \mathrm{~mm}$," Electron. Lett., vol. 27, pp. 118-120, 1991.

[44] G. V. Treyz, P. G. May, and J.-M. Halbout, "Silicon optical modulators at 1.3 micrometer based on free-carrier absorption," IEEE Electron Device Letters, vol. 12, pp. 276-278, 1991.

[45] S. M. Jackson, P.D. Hewitt, G.T. Reed, C.K. Tang, A.G.R. Evans, J. Clark, C. Aveyard, and F. Namavar, "Novel optical phase modulator design suitable for phased arrays," J. Lightwave Technol., vol. 16, pp. 2016-2019, 1998.

[46] S. M. Jackson, G. T. Reed, C. K. Tang, A. G. R. Evans, J. Clark, C. Aveyard, and F. Namavar, "Optical beamsteering using integrated optical modulators," Journal of Lightwave Technology, vol. 15, pp. 2259-2263, 1997.

[47] U. Fischer, B. Schuppert, and K. Petermann, "Integrated optical switches in silicon based on SiGe-waveguides," IEEE Photonics Technology Letters, vol. 5, pp. 785-7, 1993/07/ 1993.

[48] C. K. Tang, G.T. Reed, A.J. Wilson, and A.G. Rickman, "Low-loss, single-mode, optical phase modulator in SIMOX material," J. Lightwave Technol., vol. 12, pp. 1394-1400, October 1994.

[49] C. K. Tang, G.T. Reed, A.J. Wilson, and A.G. Rickman, "Simulation of a low loss optical modulator for fabrication in SIMOX material," in Silicon-Based Optoelectronic Materials Symposium, 12-14 April 1993, San Francisco, CA, USA, 1993, pp. 247-52.

[50] C. K. Tang and G. T. Reed, "Highly efficient optical phase modulator in SOI waveguides," Electronics Letters, vol. 31, pp. 451-452, 1995 .

[51] A. Cutolo, M. Iodice, P. Spirito, and L. Zeni, "Silicon electro-optic modulator based on a three terminal device integrated in a low-loss single-mode SOI waveguide," Journal of Lightwave Technology, vol. 15, pp. 505-518, 1997.

[52] A. Cutolo, M. Iodice, A. Irace, P. Spirito, and L. Zeni, "An electrically controlled Bragg reflector integrated in a rib silicon on insulator waveguide," Applied Physics Letters, vol. 71, pp. 199-201, 1997.

[53] A. Sciuto, S. Libertino, A. Alessandria, S. Coffa, and G. Coppola, "Design, fabrication, and testing of an integrated Si-based light modulator," Journal of Lightwave Technology, vol. 21, pp. 228-235, 2003.

[54] P. D. Hewitt, and G.T. Reed, "Improving the response of optical phase modulators in SOI by computer simulation," Journal of Lightwave Technology, vol. 18, pp. 443-450, March 2000.

[55] P. D. Hewitt and G. T. Reed, "Multi micron dimension optical p-i-n modulators in silicon-on-insulator," in Proc. Photonics West, San Jose, CA, USA, 1999, pp. 237-43.

[56] I. E. Day, S. W. Roberts, R. O'Carroll, A. Knights, P. Sharp, G. F. Hopper, B. J. Luff, and M. Asghari, "Single-chip variable optical attenuator and multiplexer subsystem integration," Anaheim, CA, United States, 2002, pp. 72-73.

[57] P. Dainesi, A. Kung, M. Chabloz, A. Lagos, Ph. Fluckiger, A. Ionescu, P. Fazan, M. Declerq, Ph. Renaud, and Ph. Robert, "CMOS compatible fully integrated Mach-Zehnder interferometer in SOI technology," IEEE Photonics Technology Letters, vol. 12, pp. 660-662, June 2000.

[58] C. E. Png, S. P. Chan, S. T. Lim, and G. T. Reed, "Optical phase modulators for MHz and GHz modulation in siliconon-insulator (SOI)," Journal of Lightwave Technology, vol. 22, pp. 1573-1582, 2004.

[59] C. A. Barrios, V. R. Almeida, R. Panepucci, and M. Lipson, "Electrooptic modulation of silicon-on-insulator submicrometer-size waveguide devices," Journal of Lightwave Technology, vol. 21, pp. 2332-9, 2003.

[60] A. Liu, R. Jones, L. Liao, D. Samara-Rubio, D. Rubin, O. Cohen, R. Nicolaescu, and M. Paniccia, "A high-speed silicon optical modulator based on a metal-oxide-semiconductor capacitor," Nature, vol. 427, pp. 615-618, 2004.

[61] L. Liao, D. Samara-Rubio, M. Morse, A. Liu, D. Hodge, D. Rubin, U. D. Keil, and T. Franck, "High speed silicon Mach-Zehnder modulator," Optics Express, vol. 13, pp. 3129-3135, 2005.

[62] K. Kajikawa, T. Tabei, and H. Sunami, "An infrared silicon optical modulator of metal-oxide-semiconductor capacitor based on accumulation-carrier absorption," Japanese Journal of Applied Physics, vol. 48, pp. 04-107, 2009.

[63] D. Andrea, "CMOS Photonics Today \& Tomorrow Enabling Technology," in Proc. OFC, 2009, San Diego, 2009.
[64] F. Y. Gardes, G. T. Reed, N. G. Emerson, and C. E. Png, "A sub-micron depletion-type photonic modulator in silicon on insulator," Optics Express, vol. 13, 2005.

[65] A. Liu, L. Liao, D. Rubin, H. Nguyen, B. Ciftcioglu, Y. Chetrit, N. Izhaky, and M. Paniccia, "High-speed optical modulation based on carrier depletion in a silicon waveguide," Optics Express, vol. 15, pp. 660-668, 2007.

[66] D. Marris-Morini, X. L. Roux, L. Vivien, E. Cassan, D. Pascal, M. Halbwax, S. Maine, S. Lavai, J. M. Fedeli, and J. F. Damlencourt, "Optical modulation by carrier depletion in a silicon PIN diode," Optics Express, vol. 14, pp. 10838$10843,2006$.

[67] C. Gunn, "CMOS Photonics for High-Speed Interconnects," Micro, IEEE, vol. 26, pp. 58-66, 2006.

[68] J. W. Park, J.-B. You, I. G. Kim, and G. Kim, "High-modulation efficiency silicon Mach-Zehnder optical modulator based on carrier depletion in a PN Diode," Opt. Express, vol. 17, pp. 15520-15524, 2009.

[69] A Narasimha et al., "An ultra low power CMOS Photonics Technology Platform for H/S Optoelectronic Transceivers at less than \$1per Gbps," in Proc. OFC, 22-25 March, San Diego, USA 2010.

[70] L. Tsung-Yang, A. Kah-Wee, F. Qing, S. Jun-Feng, X. Yong-Zhong, Y. Ming-Bin, L. Guo-Qiang, and K. Dim-Lee, "Silicon Modulators and Germanium Photodetectors on SOI: Monolithic Integration, Compatibility, and Performance Optimization," Selected Topics in Quantum Electronics, IEEE Journal of, vol. 16, pp. 307-315, 2010.

[71] N.-N. Feng, S. Liao, D. Feng, P. Dong, D. Zheng, H. Liang, R. Shafiiha, G. Li, J. E. Cunningham, A. V. Krishnamoorthy, and M. Asghari, "High speed carrier-depletion modulators with $1.4 \mathrm{~V}-\mathrm{cm} \mathrm{V} \pi \mathrm{L}$ integrated on $0.25 \mu \mathrm{m}$ silicon-on-insulator waveguides," Optics Express, vol. 18, pp. 7994-7999, 2010.

[72] D. M. Gill, M. Rasras, T. Kun-Yii, C. Young-Kai, A. E. White, S. S. Patel, D. Carothers, A. Pomerene, R. Kamocsai, C. Hill, and J. Beattie, "Internal Bandwidth Equalization in a CMOS-Compatible Si-Ring Modulator," Photonics Technology Letters, IEEE, vol. 21, pp. 200-202, 2009.

[73] S. J. Spector, M. W. Geis, M. E. Grein, R. T. Schulein, J. U. Yoon, D. M. Lennon, F. Gan, G. -R. Zhou, F. X. Kaertner, T. M. Lyszczarz, "High-speed silicon electro-optical modulator that can be operated in carrier depletion or carrier injection mode," 2008 Conference on Lasers and Electro-Optics (CLEO), May 2008

[74] S. Manipatruni, X. Qianfan, B. Schmidt, J. Shakya, and M. Lipson, "High Speed Carrier Injection 18 Gb/s Silicon Micro-ring Electro-optic Modulator," in Lasers and Electro-Optics Society, 2007. LEOS 2007. The 20th Annual Meeting of the IEEE, 2007, pp. 537-538.

[75] C. E. Png, "Silicon-on-insulator phase modulators," PhD thesis, University of Surrey, 2004.

[76] M. Soljacic, S. G. Johnson, S. Fan, M. Ibanescu, E. Ippen, and J. D. Joannopoulos, "Photonic-crystal slow-light enhancement of nonlinear phase sensitivity," J. Opt. Soc. Am. B, vol. 19, pp. 2052-2059, 2002.

[77] J. Yongqiang, J. Wei, G. Lanlan, C. Xiaonan, and R. T. Chen, "80-micron interaction length silicon photonic crystal waveguide modulator," Applied Physics Letters, vol. 87, pp. 221105-1, 2005. 
[78] L. Gu, W. Jiang, X. Chen, L. Wang, and R. T. Chen, "High speed silicon photonic crystal waveguide modulator for low voltage operation," Applied Physics Letters, vol. 90, pp. 071105-3, 2007.

[79] T. Tanabe, K. Nishiguchi, E. Kuramochi, and M. Notomi, "Low power and fast electro-optic silicon modulator with lateral p-i-n embedded photonic crystal nanocavity," Opt. Express, vol. 17, pp. 22505-22513, 2009.

[80] X. Chen, Y.-S. Chen, Y. Zhao, W. Jiang, and R. T. Chen, "Capacitor-embedded 0.54 pJ/bit silicon-slot photonic crystal waveguide modulator," Opt. Lett., vol. 34, pp. 602-604, 2009.

[81] J. Li, T. P. White, L. O'Faolain, A. Gomez-Iglesias, and T. F. Krauss, "Systematic design of flat band slow light in photonic crystal waveguides," Opt. Express, vol. 16, pp. 6227-6232, 2008.

[82] A. Brimont, P. Sanchis, and J. Marti, "Strong electro-optical modulation enhancement in a slow wave corrugated waveguide," Optics Express, vol. 17, pp. 9204-9211, 2009.

[83] L. Chen, K. Preston, S. Manipatruni, and M. Lipson, "Integrated GHz silicon photonic interconnect with micrometerscale modulators and detectors," Opt. Express, vol. 17, pp. 15248-15256, 2009.

[84] C. Batten, A. Joshi, J. Orcutt, A. Khilo, B. Moss, C. Holzwarth, M. Popovic, H. Hanqing Li Smith, J. Hoyt, F. Kartner, R. Ram, V. Stojanovic, and K. Asanovic, "Building Manycore Processor-to-DRAM Networks with Monolithic Silicon Photonics," in 16th IEEE Symposium on High Performance Interconnects, 2008, pp. 21-30.

[85] V. Stojanovic, A. Joshi, C. Batten, K. Yong-jin, and K. Asanovic, "Manycore processor networks with monolithic integrated CMOS photonics," in Lasers and Electro-Optics, 2009 and 2009 Conference on Quantum electronics and Laser Science Conference. CLEO/QELS 2009. Conference on, 2009, pp. 1-2.

[86] X. Zheng, J. Lexau, Y. Luo, H. Thacker, T. Pinguet, A. Mekis, G. Li, J. Shi, P. Amberg, N. Pinckney, K. Raj, R. Ho, J. E. Cunningham, and A. V. Krishnamoorthy, "Ultra-low-energy all-CMOS modulator integrated with driver," Opt. Express, vol. 18, pp. 3059-3070, 2010.

[87] L. Liu, J. Van Campenhout, G. Roelkens, R. A. Soref, D. Van Thourhout, P. Rojo-Romeo, P. Regreny, C. Seassal, J. M. Fedeli, and R. Baets, "Carrier-injection-based electro-optic modulator on silicon-on-insulator with a heterogeneously integrated III-V microdisk cavity," Optics Letters, vol. 33, pp. 2518-20, 2008.

[88] K. Ying-hao, C. Hui-Wen, and J. E. Bowers, "High speed hybrid silicon evanescent electroabsorption modulator," Optics Express, vol. 16, pp. 9936-41, 2008.

[89] H.-W. Chen, Y.-H. Kuo, and J. E. Bowers, "25Gb/s hybrid silicon switch using a capacitively loaded traveling wave electrode," Optics Express, vol. 18, pp. 1070-1075, 2010.

[90] M. Hochberg, T. Baehr-Jones, W. Guangxi, M. Shearn, K. Harvard, L. Jingdong, C. Baoquan, S. Zhengwei, R. Lawson, P. Sullivan, A. K. Y. Jen, L. Dalton, and A. Scherer, "Terahertz all-optical modulation in a silicon-polymer hybrid system," Nature Materials, vol. 5, pp. 703-9, 2006.

[91] R. Yiwen, G. Yangsi, H. Yijie, M. Fiorentino, M. Tan, T. Kamins, T. J. Ochalski, G. Huyet, and J. S. Harris, "Quantum-Confined Stark Effect in Ge/SiGe Quantum Wells on Si," Selected Topics in Quantum Electronics, IEEE Journal of, vol. 16, pp. 85-92.

[92] J. F. Liu et al., "Design of monolithically integrated GeSi electro-absorption modulators and photodetectors on an SOI platform," Opt. Express, vol. 15, pp. 623-628, 2007.

[93] K. Preston, S. Manipatruni, A. Gondarenko, C. B. Poitras, and M. Lipson, "Deposited silicon high-speed integratedelectro-optic modulator," Opt. Express, vol. 17, pp. 5118-5124, 2009.

[94] F. G. Della Corte, S. Rao, M. A. Nigro, F. Suriano, and C. Summonte, "Electro-optically induced absorption in Si:H/-SiCN waveguiding multistacks," Optics Express, vol. 16, pp. 7540-7550, 2008.

[95] R. S. Jacobsen, K. N. Andersen, P. I. Borel, J. Fage-Pedersen, L. H. Frandsen, O. Hansen, M. Kristensen, A. V. Lavrinenko, G. Moulin, H. Ou, C. Peucheret, B. Zsigri, and A. Bjarklev, "Strained silicon as a new electro-optic material," Nature, vol. 441, pp. 199-202, 2006.

[96] N. K. Hon, K. K. Tsia, D. R. Solli, B. Jalali, and J. B. Khurgin, "Stress-induced $\chi(2)$ in silicon - Comparison between theoretical and experimental values," in Group IV Photonics San Francisco, 2009.

[97] Q. Xu, S. Manipatruni, B. Schmidt, J. Shakya, and M. Lipson, "12.5 Gbit//s carrier-injection-based silicon microring silicon modulators," Opt. Express, vol. 15, pp. 430-436, 2007. 

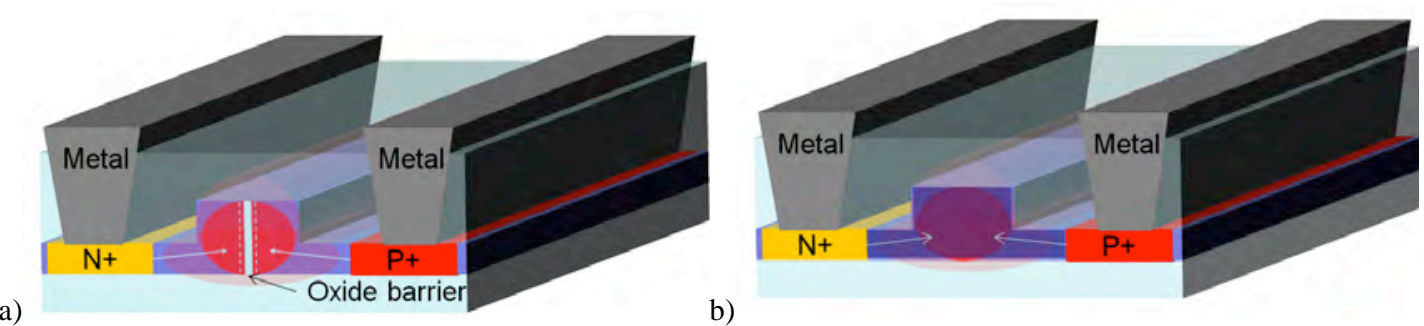

b)

c)

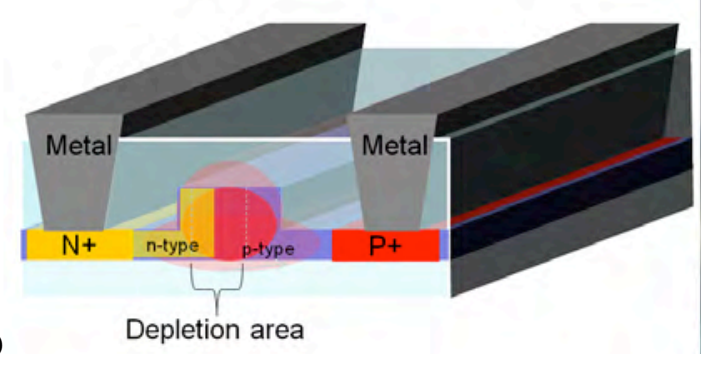

Figure 1. Cross-sectional diagrams of typical device structures which use the three different mechanisms commonly used to electrically manipulate the free carrier concentrations in plasma dispersion based silicon optical modulators a) Carrier accumulation, a thin insulating $\mathrm{SiO}_{2}$ layer is used to isolate two halves of the waveguide to form a capacitor structure. b) Carrier injection, highly doped $\mathbf{p}$ and $\mathbf{n}$ regions are separated by an 'intrinsic region' in which the waveguide is formed. Forward biasing the device causes free electrons and holes to be injected into the 'intrinsic' waveguide region. c) Carrier depletion, lightly doped p and n type regions abut in the waveguide to form a pn diode; the depletion area of the diode becomes larger with increasing reverse bias voltage. 

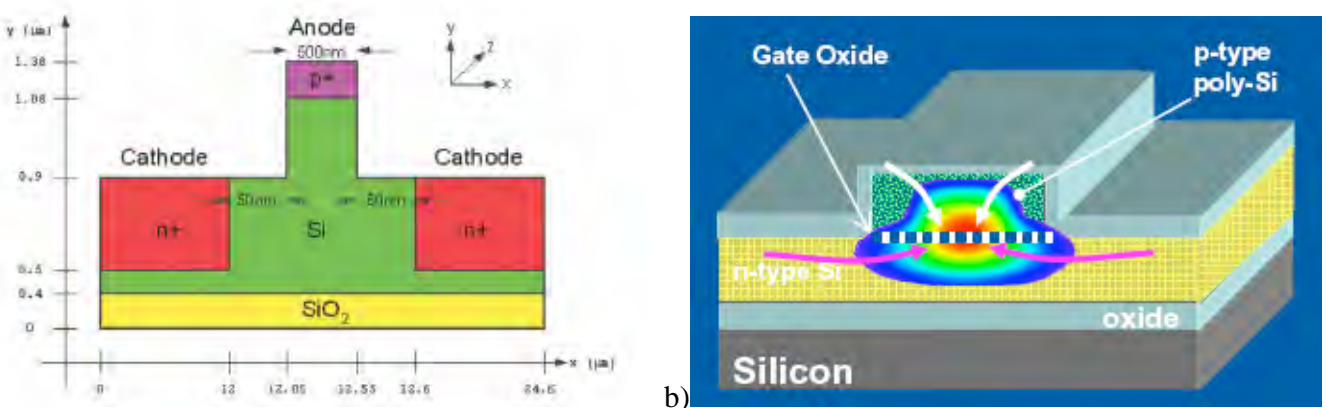

c)

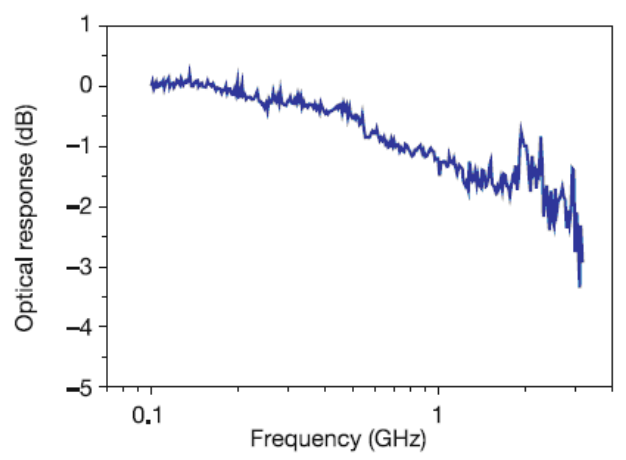

d)

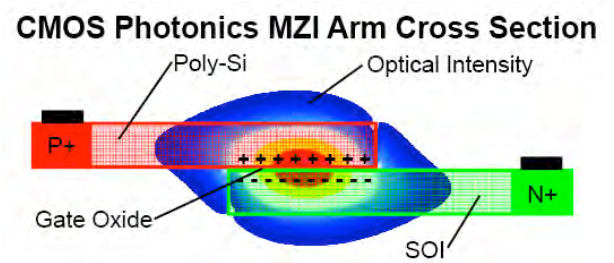

)

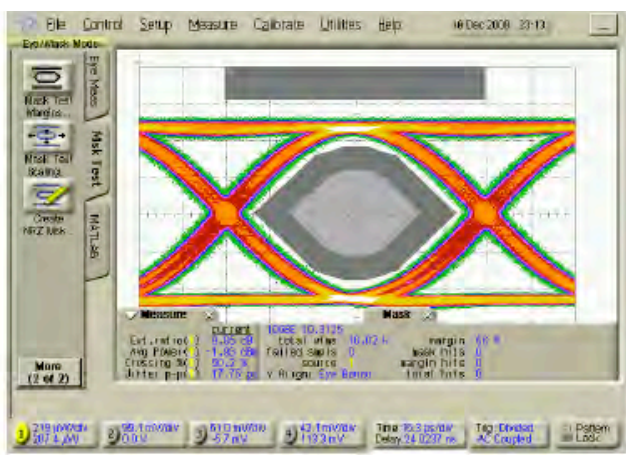

Figure 2. The first devices to propose and realise GHz modulation in silicon together with the best current Carrier Accumulation type device. a) Cross-sectional diagram of the first proposed GHz modulator which was based upon carrier injection [75]. b) Cross-secional diagram of the first experimentally realised GHz modulator based upon carrier accumulation and c) Electro-optic bandwidth of this device showing a -3dB cut-off at $\sim 3 \mathrm{GHz}[60]$. d) Cross-sectional diagram of the state of the art carrier accumulation type device and e) $10 \mathrm{~Gb} / \mathrm{s}$ eye diagram obtained from the device in (d), with $9 \mathrm{~dB}$ extinction ratio [63]. 
a)
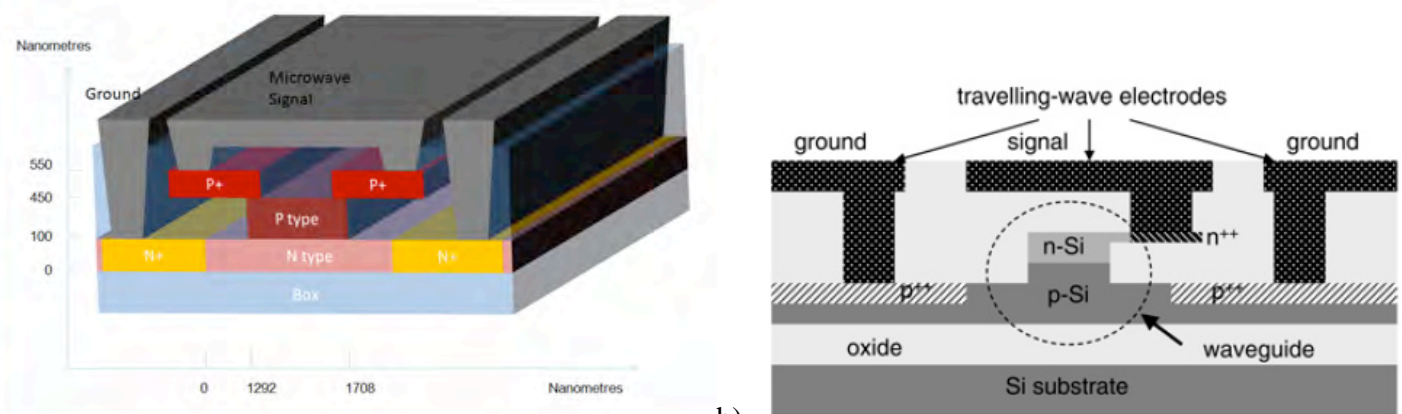

b)

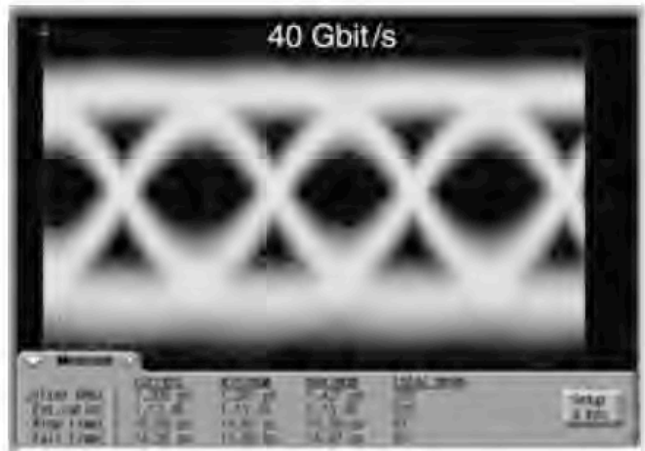

c)

Figure 3. The first proposed and the current fastest carrier depletion based silicon optical modulators. a) Cross-sectional diagram of the first proposed device which predicted an electro-optic bandwidth in excess of $50 \mathrm{GHz}$ [64]. b) Cross-sectional diagram of the fastest realised silicon optical modulator to date also based upon carrier depletion and c) eye diagram obtained from this device demonstrating data transmission at $40 \mathrm{~Gb} / \mathrm{s}$ with a $1 \mathrm{~dB}$ extinction ratio [39]. 


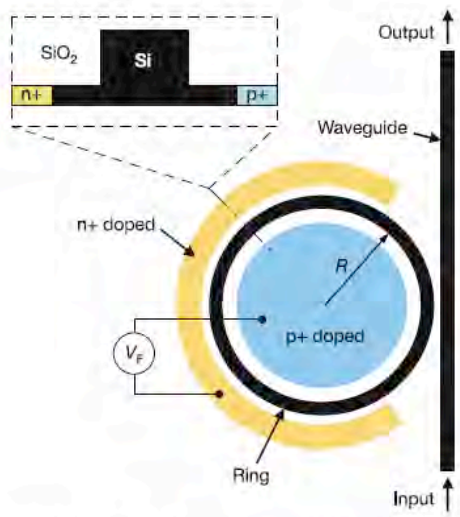

a)

Figure 4. The first silicon optical modulator employing a ring resonator structure to translate phase variations into intensity variations. a) a top down and cross-sectional diagrams of the ring resonator structure showing waveguide and doping postions and b) typical voltage induced spectral shift achieved from this device [22].

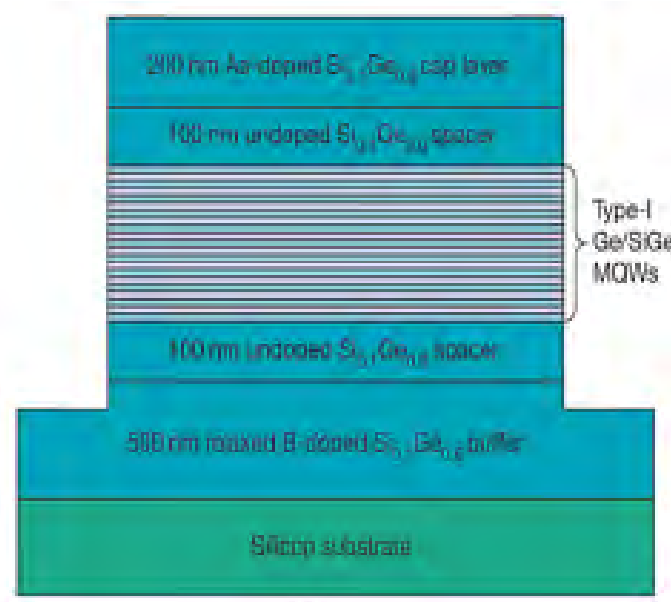

Figure 5. A possible future alternative to the plasma dispersion based silicon optical modulators is the SiGe QCSE modulator. Shown is a cross-sectional image of the p-i-n structure on silicon with $\mathrm{Ge}_{\mathrm{Si}} \mathrm{Si}_{1-x} \mathrm{Ge}_{x}$ quantum wells on a relaxed $\mathrm{Si}_{1-z} \mathrm{Ge} z$ buffer [91] 
Table 1. A comparison of the various optical modulator metrics discussed, obtained from some key example devices (after [24])

\begin{tabular}{|c|c|c|c|c|c|c|}
\hline $\begin{array}{c}\text { Modulation } \\
\text { principle }\end{array}$ & $\begin{array}{l}\text { Depletion of a } \\
\text { horizontal p-n } \\
\text { junction [61] }\end{array}$ & $\begin{array}{c}\text { Forward } \\
\text { biased } \\
\text { diode [20] }\end{array}$ & $\begin{array}{c}\text { Forward } \\
\text { biased } \\
\text { diode [74, } \\
97]\end{array}$ & $\begin{array}{c}\text { Reverse } \\
\text { biased p-n } \\
\text { junction } \\
\text { [26] }\end{array}$ & $\begin{array}{l}\text { Forward } \\
\text { biased PIN } \\
\text { diode [81] }\end{array}$ & $\begin{array}{c}\text { Reverse } \\
\text { biased p-n } \\
\text { junction } \\
\text { [24] }\end{array}$ \\
\hline Structure & MZI & MZI & Ring & Disk & Ring & Ring \\
\hline $\begin{array}{c}\text { Device } \\
\text { footprint }\end{array}$ & $\approx 1 \times 10^{4} \mu \mathrm{m}^{2}$ & $\approx 1 \times 10^{3} \mu \mathrm{m}^{2}$ & $\approx 1 \times 10^{2} \mu \mathrm{m}^{2}$ & $20 \mu \mathrm{m}^{2}$ & $\approx 1 \times 10^{2} \mu \mathrm{m}^{2}$ & $\approx 1 \times 10^{3} \mu \mathrm{m}^{2}$ \\
\hline Speed achieved & $\begin{array}{c}\text { 30 Gbps } \\
\text { (More recently } \\
\text { 40Gbps [38]) }\end{array}$ & $10 \mathrm{Gbps}$ & $>12.5 \mathrm{Gbps}$ & $10 \mathrm{Gbps}$ & $3 \mathrm{Gbps}$ & $10 \mathrm{Gbps}$ \\
\hline $\begin{array}{c}\text { Energy per bit } \\
\text { (fj/bit) }\end{array}$ & $\approx 3 \times 10^{4}$ & $\approx 5 \times 10^{3}$ & $\approx 300$ & 85 & 86 & 50 \\
\hline $\begin{array}{c}\text { Modulation } \\
\text { voltage }\end{array}$ & $6.5 \mathrm{~V}$ & $\begin{array}{l}\text { 7.6V (pre- } \\
\text { emphasis) }\end{array}$ & $\begin{array}{l}3.5 \mathrm{~V} \text { (pre- } \\
\text { emphasis) }\end{array}$ & $3.5 \mathrm{~V}$ & 0.5 & $2 \mathrm{~V}$ \\
\hline $\begin{array}{c}\text { DC } \\
\text { Modulation } \\
\text { depth and } \\
\text { insertion loss }\end{array}$ & $>20 \mathrm{~dB}, \approx 7 \mathrm{~dB}$ & $\begin{array}{l}\text { 6-10dB, } \\
12 \mathrm{~dB}\end{array}$ & $\begin{array}{l}>10 \mathrm{~dB}, \\
<0.5 \mathrm{~dB}\end{array}$ & $8 \mathrm{~dB}, 1.5 \mathrm{~dB}$ & $7 \mathrm{~dB}, 1 \mathrm{~dB}$ & $6.5 \mathrm{~dB}, 2 \mathrm{~dB}$ \\
\hline $\begin{array}{l}\text { RF Modulation } \\
\text { depth/speed }\end{array}$ & $\begin{array}{l}1 \mathrm{~dB} / 30 \mathrm{Gbps} \\
1 \mathrm{~dB} / 40 \mathrm{Gbps}\end{array}$ & - & $\begin{array}{c}\text { 8dB/16 } \\
\text { Gbps, } \\
3 \mathrm{~dB} / 18 \\
\text { Gbps }\end{array}$ & - & - & $\begin{array}{l}8 \mathrm{~dB} / 10 \\
\text { Gbps }\end{array}$ \\
\hline $\begin{array}{l}\text { Working } \\
\text { spectrum }\end{array}$ & $>20 \mathrm{~nm}$ & - & $\approx 0.1 \mathrm{~nm}$ & $\approx 0.1 \mathrm{~nm}$ & $0.2 \mathrm{~nm}$ & $\approx 0.1 \mathrm{~nm}$ \\
\hline
\end{tabular}

